\title{
INVARIANT POLYNOMIALS ON LIE ALGEBRAS OF INHOMOGENEOUS UNITARY AND SPECIAL ORTHOGONAL GROUPS
}

BY

\section{S. J. TAKIFF}

\begin{abstract}
The ring of invariant polynomials for the adjoint action of a Lie group on its Lie algebra is described for the inhomogeneous unitary and special orthogonal groups. In particular a new proof is given for the fact that this ring for the inhomogeneous Lorentz group is generated by two algebraically independent homoge ne ous polynomials of degrees two and four.
\end{abstract}

During a series of lectures given at the Indian Statistical Institute in 1965, V.S. Varadarajan proved the following result:

The ring of invariant polynomials for the adjoint action of the inhomogeneous special Lorentz group is generated by two algebraically independent homogeneous polynomials of degrees two and four.

In this paper a technique used in the above is employed to obtain another proof of this result. More generally the above ring for the inhomogeneous unitary and special orthogonal groups is essentially described. A method due to J. Rosen is used to obtain invariant polynomials for the inhomogeneous groups from those of the homogeneous groups of next higher dimension.

The author wishes to thank Professor R. Ranga Rao of the University of Illinois for suggesting the problem and making available the above mentioned lecture notes.

1. We now present some definitions, notation and preliminary results. Let $\pi: G \rightarrow G L(V)$ be a representation of a group $G$ on a finite dimensional vector space $V$. Let $S(V)$ denote the symmetric algebra of $V$ and recall that $S(V)=$ $\bigoplus_{n=0}^{\infty} S_{n}(V)$, where $S_{n}(V)$ is the subspace of homogeneous polynomials of degree $n$. We shall let $I(G, V, \pi)$ denote the algebra of polynomials in $S(V)$ invariant under the induced action of $G$ on $S(V)$; note that $I(G, V, \pi)=$ $\bigoplus_{n}^{\infty}=0\left\{S_{n}(V) \cap I(G, V, \pi)\right\}$. Now let $V^{*}$ be the dual space of $V$ and let $P(V)=$

Received by the editors July 1,1971 .

AMS 1970 subject classifications. Primary 22E43, 22E45; Secondary 15 A 72.

Key words and phrases. Invariant polynomials, inhomoge neous unitary group, inhomogene ous special orthogonal group, inhomoge ne ous Lorentz group, ad joint representation, Zariski dense, algebraically independent polynomials, contragradient representation, complexification, real form. 
$S\left(V^{*}\right)$, the algebra of polynomial functions on $V$. We define an action of $G$ on $P(V)$ as follows:

$$
\pi(g) p(v)=p\left(\pi\left(g^{-1}\right) v\right), \quad p \in P(V), v \in V, g \in G .
$$

Then denote the algebra of all $p \in P(V)$ invariant under this action by $I^{*}(G, V, \pi)$.

Now let $B$ be a nondegenerate bilinear form on $V$. We can identify $V^{*}$ with $V$ via $B$ by letting $v \rightarrow v^{*}$ where $v^{*}(w)=B(w, v), v, w \in V$. Moreover, we define $\pi^{*}: G \rightarrow G L\left(V^{*}\right)$, the representation contragradient to $\pi$, by letting

$$
\pi^{*}(g) v^{*}(w)=v^{*}\left(\pi\left(g^{-1}\right) w\right)=B\left(\pi\left(g^{-1}\right) w, v\right), \quad g \in G, \quad v, w \in V .
$$

Finally, let $V^{* *}$ be the dual space of $V^{*}$ and identify $V^{* *}$ with $V$ via $B$ by letting $v \rightarrow v^{* *}$ where $v^{* *}\left(w^{*}\right)=w^{*}(v)=B(v, w), v, w \in V$. Then the following is immediate.

Proposition 1.1. Let $\left\{v_{1}, \ldots, v_{n}\right\}$ be a bas is for $V$. Let $\phi: S(V) \rightarrow P\left(V^{*}\right)$ be defined by letting $\phi\left(p\left(v_{1}, \ldots, v_{n}\right)\right)=p\left(v_{1}^{* *}, \ldots, v_{n}^{* *}\right)$. Then $\phi$ is an isomorphis $m$ and it restricts to an isomorphism of $I(G, V, \pi)$ onto $I^{*}\left(G, V^{*}, \pi^{*}\right)$.

Now let $G$ be a connected Lie group with Lie algebra $L$ and let Ad be the adjoint representation of $G$ on $L$. Then we shall let $I(L)=I(G, L, A d)$ and $I^{*}(L)=I^{*}\left(G, L^{*}, \mathrm{Ad}^{*}\right)$. Also for $x, y \in L$ let $\operatorname{ad}(x) y=[x, y]$. Then ad extends uniquely to a derivation ad: $S(L) \rightarrow \operatorname{Der}(S(L))$, the algebra of derivations of $S(L)$ with $\operatorname{ad}(w) z=-\operatorname{ad}(z) w$ for $w, z \in S(L)$. It is easy to see that $I(L)=$ $\{w \in S(L) \mid \operatorname{ad}(x) w=0$ for all $x \in L\}=\{w \in S(L) \mid \operatorname{ad}(z) w=0$ for all $z \in S(L)\}$.

Proposition 1.2. Let $L=A \oplus T$ be a Levi decomposition of $L$ where the radical $A$ of $L$ is abelian. Then

$$
I(L)=\bigoplus_{i, j=0}^{\infty}\left\{\left(S_{i}(A) \otimes S_{j}(T)\right) \cap I(L)\right\} .
$$

Proof. Let $p \in S_{n}(L) \cap I(L)$; then $p=\sum_{j=0}^{n} p_{j}$ where $p_{j} \in S_{j}(A) \otimes S_{n-j}(T)$. Now for any $x \in L$, we have $0=\operatorname{ad}(x) p=\sum_{j=0}^{n}$ ad $(x) p_{j}$. If additionally $x \in A$ then $\operatorname{ad}(x) p_{n}=0$ and $\operatorname{ad}(x) p_{j} \in S_{j+1}(A) \otimes S_{n-j-1}(T), 0 \leq j \leq n-1$. While if $x \in$ $T$ then $\operatorname{ad}(x) p_{j} \in S_{j}(A) \otimes S_{n-j}(T), 0 \leq j \leq n$. It is then clear that the $p_{j}$ are also in $I(L)$.

The following construction is used repeatedly. Let $\eta: G \rightarrow G L(V)$ be an analytic representation of a Lie group on a finite dimensional vector space. We view $V$ as an abelian Lie group and form the semidirect product $V \times{ }_{\eta} G$ where $(v, g) \circ\left(v^{\prime}, g^{\prime}\right)=\left(v+\eta(g) v^{\prime}, g g^{\prime}\right), v, v^{\prime} \in V, g, g^{\prime} \in G$. Let $L$ be the Lie algebra of $G$ with Lie product $[,]_{L}$ and canonically identify the Lie algebra of $V$ with itself. Then the Lie algebra of $V \times_{\eta} G$ is the vector space direct sum $V \oplus L$ with Lie product $\left[v, v^{\prime}\right]=0,[x, v]=d \eta(x) v$ and $\left[x, x^{\prime}\right]=\left[x, x^{\prime}\right]_{L}$, where 
$v, v^{\prime} \in V, x, x^{\prime} \in L$ and $d \eta$ is the differential of $\eta$. We will denote this Lie algebra by $V \oplus_{\eta} L$.

If $G \subset G L(n, F)(F=\mathbf{R}$ or $\mathbf{C})$ then we shall always let $\rho: G \rightarrow G L\left(F^{n}\right)$ be as follows:

$$
\rho(A)^{t}\left(x_{1}, \cdots, x_{n}\right)=A^{t}\left(x_{1}, \cdots, x_{n}\right), \quad A \in G,{ }^{t}\left(x_{1}, \cdots, x_{n}\right) \in F^{n} .
$$

2. Recall $S O(n, \mathrm{C})=\left\{\left.A \in G L(n, \mathrm{C})\right|^{t} A A=1\right.$ and $\left.\operatorname{det} A=1\right\}$; its Lie algebra $8 \mathrm{D}(n, \mathrm{C})=\left\{M \in \mathrm{g} Y(n, \mathrm{C}) \mid M=-{ }^{t} M\right\}[3, \mathrm{p} .341]$. Assume that $n \geq 3$ and let $\mathrm{C}^{n}$ denote the complex $n$-dimensional vector space with basis $\left\{e_{1}, \ldots, e_{n}\right\}$ where $e_{j}$ is the column vector with 1 in the $j$ th row and zeros elsewhere. Now form $\mathrm{C}^{n} \times{ }_{\rho} S O(n, \mathrm{C})$ with Lie algebra $\mathrm{C}^{n} \oplus_{\rho} 8 \mathrm{D}(n, \mathrm{C})$. Let $M_{a b}$ be the $n \times n$ matrix with -1 in the $(a, b)$ position, 1 in the $(b, a)$ position and zeros elsewhere. Then $M_{a b}=-M_{b a}$ and $\left\{e_{1}, \ldots, e_{n}\right\} \cup\left\{M_{a b} \mid 1 \leq a<b \leq n\right\}$ is a basis for $\mathrm{C}^{n} \oplus_{\rho}$ $8 \mathrm{D}(n, \mathrm{C})$; moreover, for $1 \leq a, b, c, d \leq n$.

$$
\begin{aligned}
{\left[M_{a b}, M_{c d}\right] } & =\delta_{a c} M_{b d}-\delta_{b c} M_{a d}+\delta_{a d} M_{c b}-\delta_{b d} M_{c a}, \\
{\left[M_{a b}, e_{c}\right] } & =\delta_{a c} e_{b}-\delta_{b c} e_{a} \text { and }\left[e_{a}, e_{b}\right]=0,
\end{aligned}
$$

where $\delta$ is the Kronecker delta function. The following is clear.

Proposition 2.1. $p_{0}=\sum_{a=1}^{n} e_{a}^{2} \in I\left(\mathbf{C}^{n} \oplus_{\rho} 8 \mathrm{D}(n, \mathrm{C})\right)$.

We now pursue Rosen's technique [5] for obtaining invariants in $I\left(\mathbf{C}^{n} \oplus_{\rho} \overline{B D}(n, \mathbf{C})\right)$ from those in $I(B D(n,+1, \mathbf{C}))$. Let

$$
T=S\left(\mathbf{C}^{n} \oplus_{\rho} 8 \mathrm{D}(n, \mathbf{C})\right)\left[\left(-p_{0}\right)^{-1 / 2}\right]
$$

here $\left(-p_{0}\right)^{-1 / 2}$ and thus $T$ are imbedded in the algebraic closure of the quotient field of $S\left(\mathrm{C}^{n} \oplus_{\rho} \mathrm{BD}(n, \mathrm{C})\right)$. In $T$ define

$$
\begin{aligned}
\widetilde{M}_{a b} & =M_{a b}, \quad 1 \leq a, b \leq n, \\
\widetilde{M}_{a, n+1} & =-\widetilde{M}_{n+1, a}=\left(-p_{0}\right)^{-1 / 2} J_{a} \text { where } J_{a}=\sum_{b=1}^{n} e_{b} M_{a b}, 1 \leq a \leq n-1, \\
\widetilde{M}_{n+1, n+1} & =0 .
\end{aligned}
$$

Now by Proposition 2.1 ad can be uniquely extended to a derivation ad: $T \rightarrow$ Der $(T)$; and a lengthy computation shows the following to be true.

Proposition 2.2.

$$
\operatorname{ad}\left(\widetilde{M}_{a b}\right) \widetilde{M}_{c d}=\delta_{a c} \widetilde{M}_{b d}-\delta_{b c} \widetilde{M}_{a d}+\delta_{a d} \widetilde{M}_{c b}-\delta_{b d} \widetilde{M}_{c a}, \quad 1 \leq a, b, c, d \leq n+1 .
$$

Proposition 2.3. Let $q \in S\left(\mathrm{C}^{n} \oplus_{\rho} 8 \mathrm{D}(n, \mathbf{C})\right)$. Assume that ad $\left(M_{a b}\right) q=0$, $1 \leq a<b \leq n$, and $\operatorname{ad}\left(J_{a}\right) q=0,1 \leq a \leq n-1$. Then $q \in I\left(\mathbf{C}^{n} \oplus_{\rho} 8 \mathrm{D}(n, \mathrm{C})\right)$.

Proof. We need to show that ad $\left(e_{b}\right) q=0,1 \leq b \leq n$. So we compute for 
$1 \leq a \leq n, 0=\operatorname{ad}\left(J_{a}\right) q=\sum_{b=1}^{n} \operatorname{ad}\left(e_{b} M_{a b}\right) q=\sum_{b=1}^{n} e_{b}\left(\operatorname{ad}\left(M_{a b}\right) q\right)+M_{a b}\left(\operatorname{ad}\left(e_{b}\right) q\right)=$ $\sum_{b=1}^{n} M_{a b}\left(\operatorname{ad}\left(e_{b}\right) q\right)$.

Moreover by Proposition 2.1, $0=\operatorname{ad}\left(p_{0}\right) q=\sum_{b=1}^{n} 2 e_{b}\left(\operatorname{ad}\left(e_{b}\right) q\right)$. However,

$$
\begin{gathered}
\operatorname{det}\left(\begin{array}{ccccc}
-M_{12} & 0 & M_{23} & \cdots & M_{2 n} \\
-M_{13} & -M_{23} & 0 & \cdots & M_{3 n} \\
\cdot & & & & \cdot \\
\cdot & & & & \cdot \\
-M_{1 n} & -M_{2 n} & -M_{3 n} & \cdots & 0 \\
e_{1} & e_{2} & e_{3} & \cdots & e_{n}
\end{array}\right) \\
=\left(-M_{12}\right)\left(-M_{23}\right) \cdots\left(-M_{n-1, n}\right)\left(e_{n}\right)+\text { other terms } \neq 0 .
\end{gathered}
$$

Therefore $\operatorname{ad}\left(e_{b}\right) q=0,1 \leq b \leq n$.

Now for $1 \leq j \leq[(n-1) / 2]([(n-1) / 2]$ being the greatest integer less than or equal to $(n-1) / 2)$ let

$$
p_{n, 2 j}=\sum_{1 \leq a_{1}}, \cdots, a_{2 j} \leq n M_{a_{1} a_{2}} M_{a_{2} a_{3}} \cdots M_{a_{2 j-1} a_{2 j}}{ }^{M_{a_{2 j} a_{1}}}
$$

And if $n$ is even let

$$
p_{n}=\sum_{\sigma} \Delta(\sigma) M_{\sigma(1) \sigma(2)} M_{\sigma(3) \sigma(4)} \cdots M_{\sigma(n-1) \sigma(n)}
$$

where the sum is taken over all permutations $\sigma$ of the set $\{1,2, \ldots, n\}$ with $\Delta(\sigma)=1(-1)$ if the permutation $\sigma$ is even (odd). Then it is well known (see [2], [4], or [5]) that $I(Z D(n, C))$ equals $\mathrm{C}\left[p_{n, 2}, \cdots, p_{n, n-1}\right]$ if $n$ is odd and it equals $\mathrm{C}\left[p_{n}, p_{n, 2}, \cdots, p_{n, n-2}\right]$ if $n$ is even. Moreover, the polynomials $p_{n, 2}, \cdots, p_{n, n-1}\left(p_{n}, p_{n, 2}, \cdots, p_{n, n-2}\right)$ are algebraically independent over $\mathrm{C}$, for $n$ odd (even).

Finally for $1 \leq j \leq[(n-2) / 2]$ let

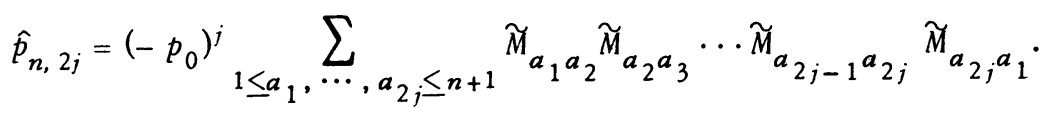

Then by Propositions 2.2 and 2.3 , the $\hat{p}_{n, 2 j}$ are in $I\left(\mathrm{C}^{n} \oplus_{\rho} 8 \mathrm{D}(n, \mathrm{C})\right)$.

3. Let $U(n)=\left\{A \in G L(n, \mathrm{C}) \mid A{ }^{t} \bar{A}=1\right\}$ with Lie algebra $u(n)=$ $\left\{M \in \mathrm{g} \backslash(n, \mathrm{C}) \mid M=-{ }^{t} \bar{M}\right\}[3, \mathrm{p} .341]$. Again assume that $n \geq 3$ and form $\mathrm{C}^{n} \times_{\rho}$ $\mathrm{U}(n)$ with Lie algebra $\mathrm{C}^{n} \oplus_{\rho} u(n)$. Now let $V$ be a complex vector space with $\operatorname{dim}_{C} V=2 n$ and let $\left\{v_{1}, \ldots, v_{2 n}\right\}$ be a basis for $V$. Let $E_{a b}$ be the $n \times n$ matrix with 1 in the $(a, b)$ position and zeros elsewhere; then $\left\{E_{a b} \mid 1 \leq a, b \leq n\right\}$ is

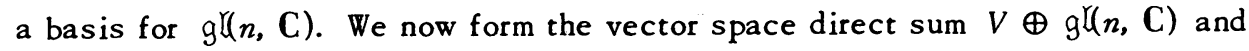
make it into a Lie algebra by letting 


$$
\begin{aligned}
{\left[E_{a b}, E_{c d}\right] } & =\delta_{b c} E_{a d}-\delta_{a d} E_{c b}, \\
{\left[E_{a b}, v_{c}\right] } & =1 / 2 \delta_{a c}\left(-v_{b}-\sqrt{-1} v_{n+b}\right)+1 / 2 \delta_{b c}\left(v_{a}-\sqrt{-1} v_{n+a}\right), \\
{\left[E_{a b}, v_{n+c}\right] } & =1 / 2 \delta_{a c}\left(\sqrt{-1} v_{b}-v_{n+b}\right)+1 / 2 \delta_{b c}\left(\sqrt{-1} v_{a}+v_{n+a}\right), \\
{\left[v_{a}, v_{b}\right] } & =0, \quad 1 \leq a, b, c, d \leq n .
\end{aligned}
$$

It is easily checked that this Lie algebra is isomorphic to the complexification of the real Lie algebra $\mathrm{C}^{n} \oplus_{\rho} \mathrm{u}(n)$. Moreover if $\pi$ is the analytic homomorphism of $G L(n, \mathrm{C})$ into $G L(V)$ such that $d \pi(M)(v)=[M, v]$ for $M \in g((n, \mathrm{C})$ and $v \in V$, then it is clear that $V \times_{\pi} G L(n, \mathrm{C})$ is a connected Lie group with Lie a lgebra $V \oplus_{\pi} g(h, \mathrm{C})$ equal to the Lie algebra just constructed. The following is immediate.

Proposition 3.1. $q_{0}=\sum_{a=1}^{2 n} v_{a}^{2} \in I\left(V \oplus_{\pi} g\lfloor(n, \mathrm{C}))\right.$.

Now let $T^{\prime}=S\left(V \oplus_{\pi} g^{Y}(n, \mathbf{C})\right)\left[\left(-q_{0}\right)^{-1 / 2}\right]$. In $T^{\prime}$ we define, for $1 \leq a, b \leq n$,

$$
\begin{aligned}
\widetilde{E}_{a b} & =E_{a b}, \quad \widetilde{E}_{a, n+1}=\left(-q_{0}\right)^{-1 / 2} K_{a}, \quad \widetilde{E}_{n+1, a}=\left(-q_{0}\right)^{-1 / 2} K_{n+a}, \\
\widetilde{E}_{n+1, n+1} & =\left(-q_{0}\right)^{-1} \sum_{a=1}^{n}\left(\sqrt{-1} v_{a}+v_{n+a}\right) K_{n+a},
\end{aligned}
$$

where

$$
K_{a}=\sum_{b=1}^{n}\left(\sqrt{-1} v_{b}+v_{n+b}\right) E_{a b} \text { and } K_{n+a}=\sum_{b=1}^{n}\left(-\sqrt{-1} v_{b}+v_{n+b}\right) E_{b a} .
$$

We see from Proposition 3.1 that ad can be uniquely extended to a derivation ad: $T^{\prime} \rightarrow \operatorname{Der}\left(T^{\prime}\right)$; and a lengthy computation establishes the following.

Proposition 3.2. $\operatorname{ad}\left(\widetilde{E}_{a b}\right) \widetilde{E}_{c d}=\delta_{b c} \widetilde{E}_{a d}-\delta_{a d} \widetilde{E}_{c b}, 1 \leq a, b, c, d \leq n+1$.

Proposition 3.3. Let $q \in S\left(V \oplus_{\pi} g .((n, \mathrm{C}))\right.$. Assume that ad $\left(E_{a b}\right) q=0$, $1 \leq a, b \leq n$, and $\operatorname{ad}\left(K_{a}\right) q=0,1 \leq a \leq 2 n$. Then $q \in I\left(V \oplus_{\pi} g((n, \mathbf{C}))\right.$.

Proof. We need to show that $\operatorname{ad}\left(v_{b}\right) q=0,1 \leq b \leq 2 n$. Now for $1 \leq a \leq n$ we compute

$$
\begin{aligned}
0 & =\operatorname{ad}\left(K_{a}\right) q=\operatorname{ad}\left(\left(\sum_{b=1}^{n}\left(\sqrt{-1} v_{b}+v_{n+b}\right) E_{a b}\right) q\right. \\
& =\sum_{b=1}^{n} E_{a b}\left(\operatorname{ad}\left(\sqrt{-1} v_{b}+v_{n+b}\right) q\right)+\left(\sqrt{ }-1 v_{b}+v_{n+b}\right)\left(\operatorname{ad}\left(E_{a b}\right) q\right) \\
& =\sum_{b=1}^{n} E_{a b}\left(\sqrt{-1} \operatorname{ad}\left(v_{b}\right) q+\operatorname{ad}\left(v_{n+b}\right) q\right) .
\end{aligned}
$$


Similarly, for $1 \leq a \leq n$,

$$
0=\operatorname{ad}\left(K_{n+a}\right) q=\sum_{b=1}^{n} E_{a b}\left(-\sqrt{-1} \operatorname{ad}\left(v_{b}\right) q+\operatorname{ad}\left(v_{n+b}\right) q\right)
$$

However,

$$
\operatorname{det}\left(\begin{array}{ccc}
E_{11} & \cdots & E_{1 n} \\
\cdot & & \cdot \\
\cdot & & \cdot \\
E_{n 1} & \cdots & E_{n n}
\end{array}\right)=E_{11} E_{22} \cdots E_{n n}+\text { other terms } \neq 0
$$

and thus $\operatorname{ad}\left(v_{b}\right) q=0=\operatorname{ad}\left(v_{n+b}\right) q, 1 \leq b \leq n$.

Now let $D$ be the matrix

$$
\left(\begin{array}{ccc}
E_{11} & \cdots & E_{1 n} \\
\cdot & & \cdot \\
\cdot & & \cdot \\
E_{n 1} & \cdots & E_{n n}
\end{array}\right)
$$

and let

$$
q_{n, j}=\operatorname{tr}\left(D^{j}\right)=\sum_{1 \leq a_{1}, \cdots, a_{j} \leq n} E_{a_{1} a_{2}} E_{a_{2} a_{3}} \cdots E_{a_{j-1} a_{j}} E_{a_{j} a_{1}}, \quad 1 \leq j \leq n,
$$

where $\operatorname{tr}()$ denotes the trace of ( ). Then it follows from [2] or [4] that $I(g)(n, \mathrm{C}))=\mathrm{C}\left[q_{n, 1}, q_{n, 2}, \cdots, q_{n, n}\right]$. Finally let

$$
\hat{q}_{n, j}=\left(q_{0}\right)^{j} \sum_{1 \leq a_{1}, \cdots, a_{j} \leq n+1} \widetilde{E}_{a_{1} a_{2}} \widetilde{E}_{a_{2} a_{3}} \cdots \widetilde{E}_{a_{j-1} a_{j}} \widetilde{E}_{a_{j} a_{1}},
$$

$1 \leq j \leq n-1$. Then by Propositions 3.2 and 3.3 we see that the $\hat{q}_{n, j}$ are in $I\left(V \oplus_{\pi} g(n, \mathrm{C})\right)$.

4. In this section we present the result from Varadarajan's lectures that we need. Let $\left(F^{n}, G, L\right)$ denote either $\left(\mathbf{R}^{n}, S O(n, \mathbf{R}), 8 \mathrm{~B}(n, \mathbf{R})\right)$ or $\left(\mathbf{C}^{n}, U(n), u(n)\right)$, with $n \geq 3$. Form $F^{n} \times_{\rho} G$ with Lie algebra $F^{n} \oplus_{\rho} L$. Now for $x=\sum_{i=1}^{n} x_{i} e_{i}, y=$ $\sum_{i=1}^{n} y_{i} e_{i} \in F^{n}$ let $\langle x, y\rangle=\sum_{i=1}^{n}\left(x_{i} \bar{y}_{i}+\bar{x}_{i} y_{i}\right)$, and note that $\langle A x, A y\rangle=\langle x, y\rangle$ for all $A \in G$. Finally let $B$ be the following bilinear form on $F^{n} \oplus L$ :

$$
B((x, M),(y, N))=\langle x, y\rangle+\operatorname{tr}\left({ }^{t} M N\right), \quad x, y \in F^{n}, \quad M, N \in L .
$$

It is clear that $B$ is nondegenerate and we can then identify $\left(F^{n} \oplus_{\rho} L\right) *$ with $F^{n} \oplus_{\rho} L$ via $B$.

Now if $x, y \in F^{n}$ then $M \rightarrow\langle M x, y\rangle$ is a real linear transformation of $L$ into $R$. Furthermore, if $\phi(N)(M)=\operatorname{tr}\left({ }^{t} M N\right), M, N \in L$, then $\phi$ is a bijective real 
linear transformation from $L$ onto $L^{*}$. Consequently, there exists a unique $\lambda(x, y) \in L$ such that $\langle M x, y\rangle=\operatorname{tr}\left({ }^{t} M \lambda(x, y)\right)$ for all $M \in L$.

Proposition 4.1. Let $x=\sum_{i=1}^{n} x_{i} e_{i}, y=\sum_{i=1}^{n} y_{i} e_{i} \in F^{n}$. Then $\lambda(x, y)$ is the matrix with $x_{j} \bar{y}_{i}-\bar{x}_{i} y_{j}$ in the $(i, j)$ position.

Proof. Let $M=\left(m_{i j}\right)$ be an arbitrary matrix in $L$ and let $\lambda(x, y)=\left(c_{i j}\right)$. Since $\operatorname{tr}\left({ }^{t} M \lambda(x, y)\right)=\sum_{i, j=1}^{n} m_{i j} c_{i j}$ and $\langle M x, y\rangle=\sum_{i, j=1}^{n} m_{i j} x_{j} \bar{y}_{i}$, we see that $c_{i j}$ $=x_{j} \bar{y}_{i}-\bar{x}_{i} y_{j}, 1 \leq i, j \leq n$.

Proposition 4.2. Let $x, y \in F^{n}, A \in G$ and $M \in L$. Then $\operatorname{Ad}(x, A)(y, M)=$ $\left(A x-A M A^{-1} x, A M A^{-1}\right)$ and $\operatorname{Ad}^{*}(x, A)(y, M)^{*}=\left(A y, \lambda(x, A y)+{ }^{t} A^{-1} M^{t} A\right) *$

Proof. The first formula follows immediately, since

$$
\begin{aligned}
\operatorname{Ad}(x, A)(y, M) & =(d / d t)\left\{(x, A) \circ \exp t(y, M) \circ(x, A)^{-1}\right\}_{t=0} \\
& =\left(A x-A M A^{-1} x, A M A^{-1}\right) .
\end{aligned}
$$

To show the second, let $z \in F^{n}$ and $N \in L$. We compute

$$
\begin{aligned}
(\operatorname{Ad} * & \left.(x, A)(y, M)^{*}\right)(z, N)=B\left(\operatorname{Ad}(x, A)^{-1}(z, N),(y, M)\right) \\
& =B\left(\left(A^{-1} z+A^{-1} N x, A^{-1} N A\right),(y, M)\right)=\left\langle A^{-1} z+A^{-1} N x, y\right\rangle+\operatorname{tr}\left({ }^{t}\left(A^{-1} N A\right) M\right) \\
& =\langle z+N x, A y\rangle+\operatorname{tr}\left({ }^{t} A^{t} N^{t} A^{-1} M\right)=\langle z, A y\rangle+\langle N x, A y\rangle+\operatorname{tr}\left({ }^{t} A^{t} N^{t} A^{-1} M\right) \\
& =\langle z, A y\rangle+\operatorname{tr}\left({ }^{t} N \lambda(x, A y)\right)+\operatorname{tr}\left({ }^{t} N^{t} A^{-1} M^{t} A\right) \\
& =B\left((z, N),\left(A y, \lambda(x, A y)+{ }^{t} A^{-1} M^{t} A\right)\right)=\left(A y, \lambda(x, A y)+{ }^{t} A^{-1} M^{t} A\right)^{*}(z, N) .
\end{aligned}
$$

Note. In the sequel we shall omit "*" and "**" from elements in dual spaces.

Let $W=\left\{(y, M) \in\left(F^{n} \oplus_{\rho} L\right) * \mid y \neq 0\right\}$ and let $U=\left\{(y, M) \in\left(F^{n} \oplus_{\rho} L\right) * \mid y=\right.$ $y_{n} e_{n}, 0<y_{n} \in R$ and $M=\left(m_{i j}\right)$ with $\left.m_{n j}=0=m_{j n}, 1 \leq j \leq n\right\}$.

Proposition 4.3. $W \subset\left\{\operatorname{Ad}^{*}(x, A) U \mid(x, A) \in F^{n} \times_{\rho} G\right\}$.

Proof. Let $(y, M) \in W$ and let $\alpha=(y, y / 2)^{1 / 2}>0$. Then $\alpha^{-1} y$ is a vector in $F^{n}$ of unit length (with respect to the usual Euclidean metric on $F^{n}$ ). Hence there exists $A \in G$ such that $A e_{n}=\alpha^{-1} y[1, \mathrm{p} .11]$, and so $A^{-1} y=\alpha e_{n}$. Now let ${ }^{t} A M^{t} A^{-1}=\left(m_{i j}\right) \in L$ and choose $x=\sum_{j=1}^{n} x_{j} e_{j} \in F^{n}$ such that $x_{j}=-m_{n j} / \alpha$, $1 \leq j \leq n-1$, and $x_{n}=-m_{n n} / 2 \alpha$. Then $\operatorname{Ad}^{*}\left(x, A^{-1}\right)(y, M)=\left(A^{-1} y, \lambda\left(x, A^{-1} y\right)+\right.$ $\left.{ }^{t} A M^{t} A^{-1}\right)=\left(\alpha e_{n}, \lambda\left(x, a e_{n}\right)^{n n}+\left(m_{i j}\right)\right) \in U$. Consequently, $(y, M) \in \operatorname{Ad}^{*}\left(x, A^{-1}\right)^{-1} U$ and so $W \subset\left\{\operatorname{Ad}^{*}(x, A) U \mid(x, A) \in F^{n} \times_{\rho} G\right\}$.

5. We now apply the results of $\S 4$. So let $x=\sum_{j=1}^{n} x_{j} e_{j}, y=\sum_{j=1}^{n} y_{j} e_{j} \epsilon$ $\mathrm{C}^{n}$, let $M, N \in \mathrm{BD}(n, \mathrm{C})$ and let

$$
B^{\prime}((x, M),(y, N))=\sum_{j=1}^{n}\left(x_{j} \bar{y}_{j}+\bar{x}_{j} y_{j}\right)+\operatorname{tr}\left({ }^{t} M N\right) .
$$


Further, for $x=\sum_{j=1}^{2 n} x_{j} v_{j}, y=\sum_{j=1}^{2 n} y_{j} v_{j} \in V$ and $M, N \in g((n, \mathbf{C})$ let

$$
B^{\prime \prime}((x, M),(y, N))=2\left(\sum_{j=1}^{n}\left(x_{j} y_{j}+x_{n+j} y_{n+j}\right)\right)+\operatorname{tr}\left({ }^{t} M N\right) .
$$

Then $B^{\prime}\left(B^{\prime \prime}\right)$ is a nondegenerate bilinear form on $\mathbf{C}^{n} \oplus_{\rho} 8 \mathrm{D}(n, \mathrm{C})\left(V \oplus_{\pi} g((n, \mathrm{C}))\right.$ and we can identify $\left(\mathrm{C}^{n} \oplus_{\rho} \mathrm{BD}(n, \mathrm{C})\right) *\left(\left(V \oplus_{\pi} \mathrm{g}^{Y}(n, \mathrm{C})\right) *\right)$ with $\mathrm{C}^{n} \oplus_{\rho} \mathrm{BD}(n, \mathrm{C})$ $\left(V \oplus_{\pi} g((n, \mathrm{C}))\right.$ via $B^{\prime}\left(B^{\prime \prime}\right)$.

Now let $W^{\prime}=\left\{(y, M) \in\left(\mathbf{C}^{n} \oplus_{\rho} 80(n, \mathbf{C})\right) * \mid 0 \neq y \in \mathbf{R}^{n}, M \in \mathrm{BD}(n, \mathbf{R})\right\}$ and let $U^{\prime}=\left\{(y, M) \in\left(\mathbf{C}^{n} \oplus_{\rho} 8 \mathrm{D}(n, \mathbf{C})\right) * \mid y=y_{n} e_{n}, 0<y_{n} \in \mathbf{R}, M=\left(m_{i j}\right)\right.$ with $m_{n j}=0=$ $\left.m_{j n}, 1 \leq j \leq n\right\}$. Moreover, let $W^{\prime \prime}=\left\{(y, M) \in\left(V \oplus_{\pi} g \backslash(n, \mathbf{C})\right) * \mid 0 \neq y \in \sum_{j=1}^{2 n} \mathbf{R} v_{j}\right.$, $M \in u(n)\}$ and let $U^{\prime \prime}=\left\{(y, M) \in\left(V \oplus_{\pi} g \backslash(n, \mathrm{C})\right) * \mid y=y_{n} e_{n}+y_{2 n} e_{2 n}, 0<y_{n}=\right.$ $y_{2 n} \in \mathbf{R}, M=\left(m_{i j}\right)$ with $\left.m_{n j}=0=m_{j n}, 1 \leq j \leq n\right\}$. Then Proposition 4.3 yields the following result.

Proposition 5.1. $W^{\prime} \subset\left\{\operatorname{Ad}^{*}(x, A) U^{\prime} \mid(x, A) \in \mathbf{C}^{n} \times_{\rho} S O(n, \mathbf{C})\right\}$ and $W^{\prime \prime} \subset$ $\left\{\operatorname{Ad}^{*}(x, A) U^{\prime \prime} \mid(x, A) \in V \times_{\pi} G L(n, \mathrm{C})\right\}$.

Corollary 5.2. Let $p, q \in I^{*}\left(\mathrm{C}^{n} \oplus_{\rho} 8 \mathrm{D}(n, \mathrm{C})\right)\left(I^{*}\left(V \oplus_{\pi} g^{Y}(n, \mathrm{C})\right)\right)$ and assume that $p\left|U^{\prime}=q\right| U^{\prime}\left(p\left|U^{\prime \prime}=q\right| U^{\prime \prime}\right)$. Then $p=q$.

Proof. By Proposition 5.1, $p-q \mid W^{\prime}=0\left(p-q \mid W^{\prime \prime}=0\right)$. Since $W^{\prime}\left(W^{\prime \prime}\right)$ is C-Zariski dense in $\left(C^{n} \oplus_{\rho} \delta \mathrm{D}(n, \mathrm{C})\right) *\left(\left(V \oplus_{\pi} g((n, \mathbf{C})) *\right)\right.$, we see that $p=q$.

Proposition 5.3. Let $p \in I^{*}\left(\mathrm{C}^{n} \oplus_{\rho} \mathrm{BD}(n, \mathrm{C})\right)$ and let $q \in I^{*}\left(V \oplus_{\pi} g \mathfrak{Y}(n, \mathrm{C})\right)$.

1. $p \mid U^{\prime} \in \mathbf{C}\left[e_{n}, p_{n-1}, p_{n-1,2}, p_{n-1,4}, \ldots, p_{n-1, n-3}\right]$ if $n$ is odd.

2. $p \mid U^{\prime} \in \mathrm{C}\left[e_{n}, p_{n-1,2}, p_{n-1,4}, \cdots, p_{n-1, n-2}\right]$ if $n$ is even.

3. $q \mid U^{\prime \prime} \in \mathrm{C}\left[v_{n}, q_{n-1,1}, q_{n-1,2}, \cdots, q_{n-1, n-2}\right]$.

Here we identify in the natural way $I^{*}(8 D(n-1, \mathrm{C}))$ and $\left.I^{*}(g)(n-1, \mathrm{C})\right)$ as subalgebras of $P\left(\left(\mathbf{C}^{n} \oplus_{\rho} \delta \mathrm{D}(n, \mathrm{C})\right) *\right)$ and $P\left(\left(V \oplus_{\pi} g(l(n, \mathrm{C})) *\right)\right.$ respectively.

Proof. By Proposition 1.2 we can assume $p \in\left(P_{a}\left(\mathrm{C}^{n *}\right) \otimes P_{b}\left(8 \mathrm{D}(n, \mathrm{C})^{*}\right)\right)$ $\cap I^{*}\left(\mathbf{C}^{n} \oplus_{\rho} 8 \mathrm{D}(n, \mathbf{C})\right)$ and $\left.q \in\left(P_{c}\left(\mathbf{C}^{n *}\right) \otimes P_{d}(g)(n, \mathbf{C}) *\right)\right) \cap I^{*}\left(V \oplus_{\pi} g((n, \mathbf{C}))\right.$. Then $p \mid U^{\prime}=\left(e_{n}\right)^{a} p^{\prime}$ and $q \mid U^{\prime \prime}=\left(v_{n}\right)^{c} q^{\prime}$ where $p^{\prime} \in I^{*}(80(n-1, \mathrm{C}))$ and $q^{\prime} \epsilon$ $\left.I^{*}(g)(n-1, \mathrm{C})\right)$. . The proposition then follows from the statements at the end $\S \$ 2$ and 3 .

Corollary 5.4. Let $n$ be odd. Then $p_{0}, \hat{p}_{n, 2}, \hat{p}_{n, 4}, \ldots, \hat{p}_{n, n-3}$ are in $I^{*}\left(\mathbf{C}^{n} \oplus_{\rho} \delta_{D}(n, \mathbf{C})\right)$ and are algebraically independent over $\mathrm{C}$. Therefore

$$
(n-1) / 2 \leq \text { tran. deg. } C\left(I^{*}\left(\mathbf{C}^{n} \oplus_{\rho} 8 \mathrm{D}(n, \mathrm{C})\right)\right) \leq(n+1) / 2 .
$$

Proof. If $Q\left(p_{0}, \hat{p}_{n, 2}, \cdots, \hat{p}_{n, n-3}\right)=0$ for $Q \in \mathbf{C}\left[X_{1}, \cdots, X_{(n-1) / 2}\right]$ then

$$
0=Q\left(p_{0}, \hat{p}_{n, 2}, \cdots, \hat{p}_{n, n-3}\right) \mid U^{\prime}=Q\left(e_{n}^{2}, p_{n-1,2}, \cdots, p_{n-1, n-3}\right) .
$$


Since $e_{n}, p_{n-1,2}, \cdots, p_{n-1, n-3}$ are algebraically independent over $\mathbf{C}$ then $Q=$ 0 and we are done.

Recall that $n \geq 3$ and let $m \geq 2$.

Proposition 5.5. Let $0 \neq p \in\left(P_{a}\left(\mathbf{C}^{2 m *}\right) \otimes P_{b}(\mathrm{zD}(2 m, \mathbf{C}) *)\right) \cap I^{*}\left(\mathbf{C}^{2 m} \oplus_{\rho} 8 \mathrm{D}(2 m, \mathbf{C})\right)$ and let $0 \neq q \in\left(P_{c}\left(V^{*}\right) \otimes P_{d}(g \backslash(n, \mathrm{C}) *)\right) \cap I^{*}\left(V \oplus_{\pi} g((n, \mathrm{C}))\right.$. Then $a, b$ and $c$ are even.

Proof. Choose a positive integer $t$ such that $a+2 t \geq b$. If $a$ is odd then

$$
\begin{aligned}
\left(\left(p_{0}\right)^{1 / 2}\left(p_{0}\right)^{t} p\right) \mid U^{\prime} & =Q\left(e_{2 m}^{2}, p_{2 m-1,2}, \cdots, p_{2 m-1,2 m-2}\right) \\
& =Q\left(p_{0}, \hat{p}_{2 m, 2}, \cdots, \hat{p}_{2 m, 2 m-2}\right) \mid U^{\prime}, \quad Q \in \mathbf{C}\left[x_{1}, \cdots, X_{m}\right] .
\end{aligned}
$$

Consequently, $\left(\left(p_{0}\right)^{2 t+1} p^{2}\right)\left|U^{\prime}=Q\left(p_{0}, \hat{p}_{2 m, 2}, \cdots, \hat{p}_{2 m, 2 m-2}\right)^{2}\right| U^{\prime}$ and so by Corollary $5.2,\left(p_{0}\right)^{2 t+1} p^{2}=Q\left(p_{0}, \hat{p}_{2 m, 2}, \cdots, \hat{p}_{2 m, 2 m-2}\right)^{2}$. But $P\left(\left(\mathbf{C}^{n} \oplus_{\rho} 8 \mathrm{D}(2 m, \mathbf{C})\right)^{*}\right)$ is a unique factorization domain and $p_{0}$ is irreducible. Thus since $p_{0}$ appears an odd (even) number of times in a factorization of $\left(p_{0}\right)^{2 t+1} p^{2}\left(Q\left(p_{0}, \hat{p}_{2 m, 2}, \cdots, \hat{p}_{2 m, 2 m-2}\right)^{2}\right)$, we have a contradiction. Hence $a$ is even and it clear that $b$ is also even. A similar argument shows that $c$ is even.

The following theorem should now be clear.

Theorem 5.6. Let $p \in\left(P_{2 a}\left(\mathbf{C}^{2 m *}\right) \otimes P_{2 b}(8 \mathrm{D}(2 m, \mathbf{C}) *)\right) \cap I^{*}\left(\mathbf{C}^{2 m} \oplus_{\rho} 8 \mathrm{D}(2 m, \mathbf{C})\right)$ and let $q \in\left(P_{2 c}\left(V^{*}\right) \otimes P_{d}(g\lfloor(n, \mathbf{C}) *)) \cap I *\left(V \oplus_{\pi} g\lfloor(n, \mathbf{C}))\right.\right.$. Let $t=$ maximum $\{0, b-a\}$ and let $s=$ maximum $\{0, d-c\}$. Then $\left(p_{0}\right)^{t} p \in$ $\mathrm{C}\left[p_{0}, \hat{p}_{2 m, 2}, \hat{p}_{2 m, 4}, \cdots, \hat{p}_{2 m, 2 m-2}\right]$ and $\left(q_{0}\right)^{s} q \in \mathbf{C}\left[q_{0}, \hat{q}_{n, 1}, \hat{q}_{n, 2}, \cdots, \hat{q}_{n, n-1}\right]$.

Remark. We note that as in the proof of Corollary $5.4, p_{0}, \hat{p}_{2 m, 2}, \cdots, \hat{p}_{2 m, 2 m-2}$ are algebraically independent over $\mathbf{C}$ and so are $q_{0}, \hat{q}_{n, 1}, \cdots, \hat{q}_{n, n-1}$.

Corollary 5.7. $I^{*}\left(\mathbf{C}^{4} \oplus_{\rho} 8 \mathrm{D}(4, \mathbf{C})\right)=\mathbf{C}\left[p_{0}, \hat{p}_{4,2}\right]$.

Proof. Let $p \in I^{*}\left(\mathbf{C}^{4} \oplus_{\rho} 8 \mathrm{~B}(4, \mathbf{C})\right) \cap P_{2 a}\left(\left(\mathrm{C}^{4} \oplus_{\rho} 8 \mathrm{~b}(4, \mathbf{C})\right) *\right)$, and choose $t$ minimal such that $\left(p_{0}\right)^{t} p \in \mathbf{C}\left[p_{0}, \hat{p}_{4,2}\right]$. Since $p$ is homogene ous then $\left(p_{0}\right)^{t} p=$ $p_{0} Q\left(p_{0}, \hat{p}_{4,2}\right)+\alpha\left(\hat{p}_{4,2}\right)^{b}$ for $Q \in \mathbf{C}\left[X_{1}, X_{2}\right], \alpha \in \mathbf{C}$, and some positive integer $b$. Now if $t \neq 0$ then $a \neq 0$; but then $p_{0}$ divides $\hat{p}_{4,2}$ in $P\left(\left(C^{4} \oplus_{\rho} 8 D(4, C)\right) *\right)$. As this is not the case we must have $t=0$ and $p \in \mathbb{C}\left[p_{0}, \hat{p}_{4,2}\right]$.

Remark. Unfortunately we cannot obtain similar corollaries for $I^{*}\left(\mathrm{C}^{2 m} \oplus_{\rho} 8 \mathrm{D}(2 m, \mathbf{C})\right), m \geq 3$, and $I^{*}\left(V \oplus_{\pi} g^{Y}(n, \mathrm{C})\right), n \geq 3$.

6. Now by (1.1) statements similar to those of (5.4), (5.6) and (5.7) can be be made for $I\left(\mathrm{C}^{n} \oplus_{\rho} \mathrm{BD}(n, \mathrm{C})\right)$ and $I\left(V \oplus_{\pi} g((n, \mathrm{C})), n \geq 3\right.$. Moreover, since $\mathrm{C}^{n} \oplus_{\rho} \mathfrak{u}(n)$ is a real form of $V \oplus_{\pi} g((n, C)$ the same can be said of $I\left(\mathbf{C}^{n} \oplus_{\rho} u(n)\right)$. In fact in (5.4), (5.6) and (5.7) we can clearly replace $\mathbf{C}^{n} \times_{\rho} S O(n, \mathbf{C})$, $\mathrm{C}^{n} \oplus_{\rho} \dot{\mathrm{z} D}(n, \mathrm{C}), V \times_{\rho} G L(n, \mathbf{C})$ and $V \oplus_{\pi} g(n, \mathbf{C})$ by $\mathbf{R}^{a+b} \times_{\rho} S O(a, b), \mathbf{R}^{a+b}$ 
$\oplus_{\rho} \mathrm{BD}(a, b), \mathrm{C}^{a+b} \times_{\rho} U(a, b), \mathrm{C}^{a+b} \oplus_{\rho} \mathrm{u}(a, b), a+b \geq 3$, since the latter are real forms of the former [3, pp. 339-355]. In particular, we see that Corollary 5.7 is true (with $p_{0}$ and $\hat{p}_{4,2}$ modified) for the inhomogeneous Lorentz group $\mathbf{R}^{4} \times{ }_{\rho}$ $S O(1,3)$ with Lie algebra $\mathbf{R}^{4} \oplus_{\rho} 8 D(1,3)$.

\section{REFERENCES}

1. C. Chevalley, Theory of Lie groups. I, Princeton Math. Series, vol. 8, Princeton Univ. Press, Princeton, N.J., 1946. MR 7, 412.

2. B. Gruber and L. O'Raifeartaigh, $S$ theorem and construction of the invariants of the semisimple compact Lie algebras, J. Mathematical Phys. 5 (1964), 1796-1804. MR 30 \# 195.

3. S. Helgason, Differential ge ometry and symmetric spaces, Pure and Appl. Math., vol. 12, Academic Press, New York, 1962. MR 26 \#2986.

4. L. O'Raifeartaigh, Lectures on local Lie groups and their representations, The Institute of Mathematical Sciences, Madras, 1964.

5. J. Rosen, Construction of invariants for Lie algebras of inhomogeneous pseudoorthogonal and pseudo-unitary groups, J. Mathematical Phys. 9 (1968), 1305-1307. MR 38 \#275.

DEPARTMENT OF MATHEMATICS, MICHIGAN STATE UNIVERSITY, EAST LANSING, MICHIGAN 48823 